Sammlung wurden folgende anderweitige Objekte (Dias, Bücher etc,) geschenkt; vom Museum Rietberg, Zürich: 1 elektrische Trockenpresse für Photos und Photokopien; vom Zoologischen Institut der Universität Zürich: 110 Diapositive aus Indonesien aus dem Nachlaß von Dr. Gustav Schneider, Hefte des «Bulletin de l'Institut égyptien 1940-1955, 3 Hefte "Acta tropica»; vom Geographischen Institut der Universität Zürich: 9 Hefte der Zeitschrift "Afrika», 2 Bände Barrados: orfevreria columbiana; von der Zentralbibliothek Zürich: 6 Bücher völkerkundlichen Inhalts, $10 \mathrm{Hefte}$ ethnographischer Zeitschriften; von Prof. Dr. E. Ackerknecht, Zürich: 15 völkerkundliche Werke (aus dem Nachlaß von Dr. med. Sigrist in Pura); von Prof. Dr. Karl Suter, Zürich: 1 Buch von J. Gabus; von Prof. Dr. Emil Abegg, Zürich: 2 Bücher von W. Schmidt und v. Mzile; von der Stiftung “Jubiläumsspende für die Universität Zürich»: 1 elektrische Graviermaschine für Fr. 1300.—.

Anschaffungen. Die Sammlungsbestände wurden durch folgende Ankäufe ergänzt: Afrika : 11Objekte : Eulenmaske der Bobo, Elfenbeinküste (franz. Sudan); Maske der Vati, Vey oder Vei (Westsudan); Stülpmaske der Ibo (Kamerun); verzierte Kalebasse und 4 Bronze-Armringe aus franz. Westafrika, besticktes graues Häuptlingsgewand (Tobe) und hölzerner viergesichtiger Shangostab der Yoruba, (Nigeria); Malerei auf Ziegenleder mit Gerichtsszene aus Abessinien; Amerika: 10 Objekte: Kleiner Goldfrosch aus Panama, 2 Tonfiguren aus Alt-Mexiko, (Flötenspieler, Nayarit und weibliches Figürchen, Tlatilco), sowie eine Tonmaske, kleine männliche Grünsteinfigur aus Costarica. Aus Peru stammen: Goldpincette, Bronze-Mittelstück einer Speerschleuder, polychrome Tonfigur eines Condor (Nasca), Tonpfeife mit Füchsleinfigur und aus Ecuador menschliche Figur. Asien: 7 Objekte. 5 japanische Votiv-Tafeln (Ema's), 2 altchinesische Jade-Objekte (Zikade und Wentschung-Ahnenfigürchen aus der Zeit der Han-Dynastie). Australien: 1 Objekt. Flache Muschelschale von Meleagrina, mit eingeritzten geometrischen Ornamenten, dient angeblich als Liebeszauber und Schutzmittel gegen Krankheiten (Fig. 3).

Tausch. Von Prof. H. Baldus vom Museo Paulista in Sao Paolo wurden gegen 1 afrikanischen Nagelfetisch (Loango) und 2 Schweizermasken eingetauscht: 2 seltene mit Rindenstoff überzogene Tierschädelmasken der Tukuna (Fig. 4) und 5 bemalte Tonpuppen der Karajà-Indianer. Von Prof. A. Steinmann wurden gegen ein rundes Sawan-Kalokgefäß eingetauscht: 1 blauweißer chinesischer "Gendih" aus dem 17. Jahrhundert; 1 kleines blauweißes Ming-Väschen mit Hirschmotiven gegen 1 senffarbiges Sung-Schälchen. Die Geographisch-Ethnographische Gesellschaft Zürich überwies der Sammlung für Völkerkunde wie bisher einen jährlichen Beitrag von Fr. 500, wofür ich ihr den verbindlichsten Dank aussprechen möchte.

Der Vorsteher: ALFRED STEINMANN

\title{
ZUR LANDESKUNDLICHEN DOKUMENTATION DER SCHWEIZ RUDOLF TANK
}

In den Jahren 1892 bis 1945 erschien die Bibliographie der schwcizcrischen Landeskunde, unter Mitwirkung der Bundesbehörde, eidgenössischer und kantonaler Amtsstellen und zahlreicher Gelehrter hrg. von der Centralkommission für schweizerische Landeskunde. Das Werk verzeichnet in 89 Teilbibliographien die Buch- und Zeitschriftenliteratur aller $Z$ weige der Wissenschaften, soweit diese mit der Schweiz in Beziehung stand. Nicht berücksichtigt wurden die Geschichte und die Technik. Diese erste nationale Bibliographie, von der der größte Teil vor dem ersten Weltkrieg erschien, war retrospektiv gedacht und blieb unvollendet. Erst die Gründung der Schweizerischen Landesbibliothek im Jahre 1895 ermöglichte die Herausgabe einer laufenden nationalen Bibliographie. Die neue Bibliothek erhielt die Aufgabe, Helvetica i. w. S. zu sammeln, d. h. in der Schweiz erscheinende Veröffentlichungen, von Schweizern oder von in der Schweiz niedergelassenen Ausländern verfaßte sowie Schweizer oder die Schweiz betreffende Publikationen in- oder ausländischer Herkunft. Die Neuerwerbungen wurden im seit 19ol erstmals erscheinenden «Bibliographischen Bulletin der Schweizerischen Landesbibliothek - Bulletin bibliographique de la Bibliothèque nationale suisse» angezeigt, das seit 1915 und 1943 unter Änderung des Titels in «Das Schweizer Buch - Le livre suisse - Il libro svizzero» Erweiterung erfuhr. Als Mehrjahresverzeichnis erschien das «Systematische Verzeichnis der Schweizerischen oder die Schweiz betreffenden Veröffentlichungen», ergänzt durch einen Ortsund Personenkatalog. Perioden: 19o1-192o, 1921-193o, 1931-194o, 1941-1947.) Abgelöst wurde das Systematische Verzeichnis durch das «Schweizer Bücherverzeichnis» (1. Autoren-, 2. Sachreihe), erstmals hrg. für die Periode 1948-195o und fortgesetzt in Fünfjahresverzeichnissen (1951-1955) zusammen mit dem neuen «Schweizer Zeitschriftenverzeichnis». Außer der Nationalbibliographie gibt die Schweizerische Landesbibliothek folgende Jahres-Schrifttumsverzeichnisse heraus: «Bibliographie der schweizerischen Amtsdruckschriften», seit 1947. — «Bibliographia scientiae naturalis Helvetica», erstmals 1927 (Berichtsjahr 1925), erschien vor 1950 (1948) u.d. T. Bibliographie der schweizerischen naturwissenschaftlichen und, seit 1942 (1940), geographischen Literatur - Bibliographie der Schweizergeschichte, erstmals 1913, seit dem Jahr 
1927 mit kurzem Unterbruch in der Schweizerischen Landesbibliothek bearb., bis 1958 hrg. von der Allgemeinen geschichtsforschenden Gesellschaft der Schweiz. Sie bildet die periodische Fortsetzung der «Bibliographie zur Schweizergeschichte, enthaltend die selbständig erschienenen Druckwerke...»Bd. 1 bis Ende 1912, Bd. 2 und 3 bis Ende 1913, bearb. von H. Barth, und des «Schweizergeschichtlichen Repertoriums über die in Zeit- und Sammelschriften der Jahre 1812189o, 1891-19oo und 19o1-1912 erschienenen Aufsätze und Mitteilungen schweizergeschichtlichen Inhalts». - «Jahresverzeichnis der schweizerischen Hochschulschriften», hrg. von der Universitätsbibliothek Basel. Redaktion durch die Schweizerische Landesbibliothek ab Jg. 57, 1954. Als weitere wichtige schweizerische Amtsstellen, die die landeskundliche Dokumentation pflegen sind zu erwähnen: das Eidgenössische statistische Amt in Bern. Dieses bearbeitet u.a. die «Schweizerische Bibliographie für Statistik und Volkswirtschaft», welche von der Schweizerischen Gesellschaft für Statistik und Volkswirtschaft herausgegeben wird. Sie erschien erstmals 1938 (Berichtsjahr 1937). Die Abteilung für Hydrologie der Versuchsanstalt für Wasserbau und Erdbau an der Eidgenössischen Technischen Hochschule, Zürich bearbeitet die «Hydrologische Bibliographie der Schweiz», hrg. von der Hydrol. Kommission der Schweizer. naturforsch. Gesellschaft, der Gletscherkommission der Schweizer. naturforsch. Gesellschaft und der Eidgenössischen Kommission für Schnee- und Lawinenforschung. Erschienen sind die Hefte 1939/47, 1948/52, 1953/55, in Druck ist das Heft 1956/58. Die Schweizcrische Mcteorologische Zentralanstalt in Zürich veröffentlicht jährlich zweimal, seit 1946, die «Bibliographie météorologique suisse». Die Titel werden auch in einem Sonderkatalog der Bibliothek der MZA nachgewiesen.

Die allgemein-wissenschaftlichen Kantonsbibliotheken sammeln alle mehr oder weniger systematisch die landeskundlichen Veröffentlichungen über ihr Kantonsgebiet und weisen sie z.T. auch in eigenen alphabetischen und vielfach auch geographischen Katalogen nach. Die Zeit und die zur Verfügung stehenden Arbeitskräfte erlauben ihnen jedoch nicht, sich besondern Dokumentationsaufgaben zuzuwenden. Das Sammeln und der Nachweis unselbständiger landeskundlicher Publikationen wird daher vor allem durch die kantonalen und regionalen historischen Vereinigungen besorgt meist, auf Grund der Bestände und unter Mitarbeit der Kantonsbibliotheken.

Bei der Nennung der Institutionen, die sich mit der Herausgabe landeskundlicher Schrifttumsverzeichnisse befassen, müssen wir uns auf diejenigen Gesellschaften beschränken, deren Bibliographien eine gewisse Vollständigkeit anstreben und, falls sie ihr Erscheinen eingestellt haben, größere Zeitspannen umfassen. Eine wertvolle Zusammenstellung, besonders was selbständige und versteckte Fachschrifttumsverzeichnisse und Lokalbibliographien betrifft, bildet das «Verzeichnis landeskundlicher Bibliographien der Schweiz» von E. Winkler in den Mitteilungen der Geogr.-Ethnogr. Gesellschaft in Zürich, 31, 1941/43, S. 150-158. Die Geographisch-Ethnographische Gesellschaft in Zürich veröffentlichte in den Bänden 2o, 1919/20 bis 4o, 194o/41 ihrer Mitteilungen eine sachlich geordnete «Geographische Bibliographie der Schwciz». Sie wurde abgelöst als neue Abteilung in der von der Schweizerischen Landesbibliothek hrg. Bibliographie der schweizerischen naturwissenschaftlichen Literatur, seit Jg. 16, 1940 (1942).

Die Appenzellische gemeinnützige Gesellschaft veröffentlicht in den «Appenzeller Jahrbüchern» regelmäßig ein Verzeichnis der «Schriften, die das Land Appenzell betreffen»; seit 19o4 ist das Verzeichnis umfassender als in früheren Jahren. Der Historische Verein Appenzell bringt im «Innerhoder Geschichtsfreund》 seit 1955 - H. 3 eine Bibliographie der Veröffentlichungen betreffend die Geschichte des Halb-Kantons Appenzell Inner-Rhoden. Mit Unterstützung durch die Basler Universitätsbibliothek erscheint seit 1920 die sachlich geordnete «Basler Bibliographie». Von 1920 bis 1936 wurde sie im «Basler Jahrbuch» veröffentlicht, seither (Bd. $36,1937)$ in der «Basler Zeitschrift für Geschichte und Heimatkunde». In den Jahren 1911 (Berichtsjahre 1900-1911), 1925 (1911-1925), 1933 (1925-1933), 1936 (1933-1935), 1940 (19351939) und 1942 (1939-1942) erschien im "Tätigkeitsbericht der Naturforschenden Gesellschaft Baselland» eine systematische «Bibliographie der auf das Gebiet des (Halb-) Kantons Basclland bezüglichen naturwissenschaftlichen und (seit 1933) heimatkundlichen Literatur». Das «Neue Berner Taschenbuch» enthielt von 1918-1934 eine «Bibliographie zur Geschichte des Kantons Bern (1928 ff ... zur Geschichte und Volkskunde). In der «Schulpraxis» Jg. 46, 1956/57, H. 10/11 veröffentlichte R. Marti-Wehren ein «Verzeichnis heimatkundlicher Arbeiten aus dem Bernbiet (alter Kantonsteil [d.h.ohne Jura]). Nicht eingeschlossen sind die Lokalgeschichte, Pflanzengeographie, Geologie, Paläontologie und Urgeschichte. In den «Freiburger Geschichtsblättern», hrg. vom deutschen geschichtsforschenden Verein des Kantons Freiburg finden wir in den Jahren 1894-1904 u. 1908 eine «Bibliographie der Freiburgcr Literatur für die Jahre 1893 bis 1908. Die Société d'histoire et d'archéologie de Genève bringt in ihrem Bulletin seit 1948 eine «Chronique bibliographique». Im «Bündnerischen Monatsblatt» 1914 bis 1923 finden wir ein «Verzeichnis der (grau) bündnerischen Literatur». Im Jahre 1938 gab die Ligia romontscha mit Unterstützung durch die Kantonsbibliothek Graubünden die «Bibliographia retoromontscha» heraus, eine Bibliographie des gedruckten bündnerromanischen Schrifttums von den Anfängen bis zum Jahre 1930. 1956 folgte ein Nachtrag für die Jahre 1931-1952. Die Kantonsbibliothek Graubünden in Chur führt seit ungefähr 1950 eine Dokumentation von Zeitschriftenarti- 
keln über Bündner und Graubünden. - Im «Geschichtsfreund», den Mitteilungen des Historischen Vereins der fünf Orte (= Kantone) Luzern, Uri, Schwyz, Unterwalden ob und nid dem Wald und Zug erscheint seit 1948 die «Bibliographie der 5 Orte». Je ein Vertreter der beteiligten Kantone redigiert den Beitrag seines Gebietes. Schon in den Jahren 1880-1923 des Geschichtsfreund finden wir einen Abschnitt «Literatur der 5 Orte», allerdings nicht nach Kantonen geordnet. Das «Neujahrsblatt des Historischen Vereins des Kantons St. Gallen» enthält seit 1880 eine jährliche Übersicht über die «St. Gallische Literatur»; ab 1952 werden darin auch Zeitschriftenartikel berücksichtigt. - Die «Bibliographie zur Schaffhauser Geschichte und Kunstgeschichte» erscheint seit 1936 in den «Beiträgen zur vaterländischen Geschichte», hrg. vom Historisch-antiquarischen Verein des Kantons Schaffhausen. - Seit 1928 wird im «Jahrbuch für solothurnische Geschichte» eine «Bibliographie der solothurnischen Geschichtsliteratur veröffentlicht, hrg. vom Historischen Verein des Kantons Solothurn. - Im Jahre 1944 verfaßte H. Meister in Form einer Diplomarbeit der Ecole de bibliothécaires in Genf die "Bibliographie des publications tessinoises de 1900 à 1930. Das systematisch aufgebaute Schriftumsverzeichnis berücksichtigt nur die selbständigen Veröffentlichungen. Die Biblioteca cantonale in Lugano beabsichtigt, eine jährlich erscheinende «Bibliografia ticinese» zu veröffentlichen. Der erste Band, umfassend die selbständigen und unselbständigen Publikationen des Jahres 1957, soll im Jahr 1961 erscheinen. - In den «Blättern zur Walliser Geschichte» hrg. vom Geschichtsforschenden Verein von Oberwallis, veröffentlicht L. CARLEN seit 1951 eine «Bibliographie der Walliser Geschichte». - Die Stadtbibliothek Winterthur bearbeitet die «Bibliographie zur Orts- und Personengeschichte von Winterthur. Tößtal, Weinland». Sie erscheint alle zehn Jahre als «Neujahrsblatt der Stadtbibliothek Winterthur: 1932 (Berichtsjahre 1921-1930). 1942 (1931-1940), 1952 (1941-1950). Die Bibliographie wird in Form eines Zettelkatalogs laufend nachgeführt. - Im «Zürcher Taschenbuch» erscheint seit 1878 eine geographisch und sachlich geordnete «Bibliographie der Geschichte, Landes- und Volkskunde von Stadt und Kanton Zïrich». Die Titel werden auch in der Zentralbibliothek Zürich in deren Sonderkatalog der landeskundlichen Veröffentlichungen über Kanton und Stadt nachgewiesen.

\section{PAUL VOSSELER ZUM 70. GEBURTSTAG}

Am 2o. März 1960 trat Prof. Dr. Paul Vosseler, Vorsteher des Geographischen Institutes der Universität Basel, in das siebente Jahrzehnt seines Lebens ein. Eine angriffige, unentwegte Schaffensfreude prägte schon sein Studium; sie ist ihm in all den Jahren eigen geblieben, in denen er an Universität und Schule, als Forscher und Lehrer ein gerüttelt Maß von Arbeit bewältigte. Vosseler zählte zu den ersten Studenten des 1912 gegründeten geographischen Instituts der Basler Universität. Nach Studiensemestern in Lausanne und Berlin promovierte er 1917 mit seiner Arbeit über die Morphologie des Aargauer Tafeljuras, welche, angeregt durch seinen Lehrer G. Braun und die morphologischen Ideen von W. M. Davis, die Formentwicklung des nordöstlichen Juras aufhellt. Diese Untersuchung baute er zu einer wegweisenden Monographie des Aargauer Juras aus, mit welcher er sich 1926 die erste von der Basler Universität verliehene Venia docendi in Geographie erwarb. Bald wurde ihm ein Lehrauftrag länderkundlicher Richtung erteilt, und 1936 erfolgte die Ernennung zum außerordentlichen Professor. 1947 wurde ihm die Leitung des geographischen Instituts der Universität übertragen.

Das Verzeichnis der wissenschaftlichen Veröffentlichungen PAUL Vosselers 1 dokumentiert nicht nur eine hervorragende wissenschaftliche Leistung, sondern darüber hinaus seine Vielseitigkeit, welche Ausdruck der breiten Ausbildungsbasis, der weiten Interessenspannung und der auf ausgedehnten Studienreisen in Europa und Nordafrika erworbenen eigenen Anschauung ist. Morphologischen Fragen sind neben der Promotionsarbeit u. a. die Studien über die Schichtstufenlandschaft (1927), sodann verschiedene Arbeiten im Anschluß an die Studienreisen in der Iberischen Halbinsel (1928) und ein Aufsatz über die Schaffhauser Landschaft (1944) gewidmet. Seine Habilitationsschrift bezeugt, $d a \beta$ sich VosseLer früh auch umfassend mit dem anthropogeographischen Problemkreis befaßt; in der Folge verfaßte er für das Handbuch von FRÜH den Abschnitt über die Siedlungen (1930) und zahlreiche Aufsätze über die Städte der Schweiz, die ländlichen Siedlungen des Juras, die Bauernhausformen namentlich der Südschweiz und über die Formen des Stadthauses in ihrem Zusammenhang mit den ländlichen Bauformen u. a. Schon seine ganzheitlich orientierte Monographie über den Aargauer Jura beweist, da $B$ jedoch Paul Vosseler das Endziel allen wissenschaftlich-geographischen Bemühens nicht aus den Augen verliert, die länderkundliche Synthese. Für das Klute'sche Handbuch verfaßte er eine Geographie der Schweiz (1933), zum Früh steuerte er die Beiträge über das Wallis und die Romanischen Alpen, für «Die Erde»(Bern) die Aufsätze über die Britischen Inseln, Polen und Rumänien (1954) bei. Durch seine Untersuchungen und die Arbeiten seiner Schüler förderte er entscheidend die Kenntnisse des Juras und der Südschweiz. Bedeutend ist die Zahl

1 Siehe Regio Basiliensis I/2, 1960, S. 58-6o. 